\title{
The Application of Minimal Length in Klein-Gordon Equation with Hulthen Potential Using Asymptotic Iteration Method
}

\author{
Isnaini Lilis Elviyanti, Beta Nur Pratiwi, A. Suparmi (D), and C. Cari \\ Physics Department, Graduate Program, Sebelas Maret University, Indonesia \\ Correspondence should be addressed to A. Suparmi; soeparmi@staff.uns.ac.id
}

Received 28 February 2018; Revised 13 April 2018; Accepted 16 May 2018; Published 2 July 2018

Academic Editor: Yao-Zhong Zhang

Copyright ( 2018 Isnaini Lilis Elviyanti et al. This is an open access article distributed under the Creative Commons Attribution License, which permits unrestricted use, distribution, and reproduction in any medium, provided the original work is properly cited.

The application of minimal length formalism in Klein-Gordon equation with Hulthen potential was studied in the case of scalar potential that was equal to vector potential. The approximate solution was used to solve the Klein-Gordon equation within the minimal length formalism. The relativistic energy and wave functions of Klein-Gordon equation were obtained by using the Asymptotic Iteration Method. By using the Matlab software, the relativistic energies were calculated numerically. The unnormalized wave functions were expressed in hypergeometric terms. The results showed the relativistic energy increased by the increase of the minimal length parameter. The unnormalized wave function amplitude increased for the larger minimal length parameter.

\section{Introduction}

The relativistic effect gives the correction in the nonrelativistic quantum mechanics by applying the strong potential field in the particles dynamic. The particles dynamic in relativistic effect can be described by using Klein-Gordon equation, particularly for a zero spin particle. The Klein-Gordon equation is formed by two potentials coupling which are the four-vector potential $(V(r))$ and scalar potential $(S(r))$. From these two potentials, the Klein-Gordon equation has two framework conditions, which are as follows: if the scalar potential was equal to vector potential $(S(r)=V(r))$ and if the scalar potential was equal to minus vector potential $(S(r)=$ $-V(r))$. This condition appears in nuclear and high energy physics problem [1-3]. Some of researchers have investigated both these conditions with the certainty vector potential. The main case of that research was how to reduce the KleinGordon equation into the Schrodinger-like equation, so we can solve it by using the certainty suitable methods. The methods which are usually used are such as Supersymmetric Quantum Mechanics (SUSY) [4], Nikiforov-Uvarov [1, 5], and Asymptotic Iteration Method [6, 7]. Various potentials are used to explain the dynamic particle, such as the harmonic potential [8], Makarov potential [2], Hulthen potential [1, 5],
Kratzer potential [6], and Trigonometric Poschl-Teller potential [7].

The particles dynamic in quantum mechanics corresponds to the position and momentum of particles. And, as we have known, the study of commutation relations between position and momentum operators is explained using Heisenberg uncertainty principle [9], which is given by

$$
[\widehat{X}, \widehat{P}] \geq i \hbar
$$

where $\widehat{X}$ is position operator, $\widehat{P}$ is momentum operator, $i$ is imaginer number, and $\hbar=h / 2 \pi$ with $h$ being Planck constant. The presence of a quantum gravity on quantum mechanics has the consequence of the existence of a minimal observable distance on the order of the Planck length. Therefore the Heisenberg uncertainty principle gets additional correction due to the presence of a quantum gravity, which is well known as Generalized Uncertainty Principle (GUP) [10, 11], given by

$$
[\widehat{X}, \widehat{P}] \geq i \hbar\left(1+\alpha_{M L} P^{2}\right)
$$

where $\alpha_{M L}$ is minimal length parameter that has value $0 \leq$ $\alpha_{M L} \leq 1$ and $P$ is magnitude of the momentum [11]. When the energy is much smaller than the Planck mass, $\alpha_{M L}$ goes to zero and we recover Heisenberg uncertainty principle [12]. 
In 2009, Jana and Roy have solved Klein-Gordon equation in the presence of minimal length for scalar potential was equal to vector potential using Algebraic Approach [13]. The Klein-Gordon equation in the presence of a minimal length is solved by using Feynman for scalar potential was equal to vector potential [14]. In addition, the hypergeometric method is used to solve Klein-Gordon equation using hyperbolic cotangent potential [15] and Asymptotic Iteration Method in trigonometric cotangent potential $[16,17]$.

In this paper, we solved the minimal length formalism in radial part of Klein-Gordon equation for the condition $S(r)=$ $V(r)$ with Hulthen potential by using approximate solution. The approximate solution is used by Chabab et al. to solve the Bohr Mottelson Hamiltonian in the presence of a minimal length formalism by introducing the new wave function [11]. The minimal length formalism in the Klein-Gordon equation is reduced into second-order differential equation. The relativistic energy equation and wave functions of KleinGordon equation are obtained by using Asymptotic Iteration Method.

The study is organized as follows. In Section 2, the approximate form of Klein-Gordon equation within minimal length formalism is presented. The Hulthen potential is introduced in Section 3. We describe Asymptotic Iteration Method in Section 4. The result and discussion are presented in Section 5. At last, conclusion is given in Section 6.

\section{Approximate Form of Klein-Gordon Equation within Minimal Length Formalism}

Generalized Uncertainty Principle is called the minimal length that is deformed from the commutation relations between position and momentum operators in quantum mechanics. In (2), the commutation relations can be rewritten as follows $[9,12]$ :

$$
\begin{aligned}
\widehat{X}_{i} & =\widehat{x}_{i} \\
\widehat{P}_{i} & =\left(1+\alpha_{M L} \widehat{p}^{2}\right) \widehat{p}_{i}
\end{aligned}
$$

where $\widehat{P}_{i}$ and $\widehat{p}_{i}$ are momentum operators at high and low energy, respectively. The magnitude of $\widehat{p}_{i}$ is expressed by $p$. The occurrence of minimal length is in string theory, black hole, quantum gravity, and noncommutative geometry, which yield new correction to Heisenberg uncertainty principle and imply a finite minimal uncertainty in position measurements, e.g., at the Planck scale [14].

The general Klein-Gordon equation with scalar potential $S(r)$ and vector potential $V(r)$ is given as

$$
\begin{aligned}
(E & -V(r))^{2} \psi(r, \theta, \varphi) \\
& =\left[P^{2} c^{2}+\left(M_{o} c^{2}+S(r)\right)^{2}\right] \psi(r, \theta, \varphi)
\end{aligned}
$$

where $E$ is relativistic energy and $M_{o}$ is rest mass. By setting $S(r)=V(r)$ in (5) and substituting (4) into (5), with $\widehat{p}=$ $-i \hbar \nabla$ and $c=\hbar=1$ (natural unit), we have

$$
-\left(\Delta-2 \alpha_{M L} \Delta^{2}\right) \psi(r, \theta, \varphi)
$$

$$
-\left(\begin{array}{c}
E^{2}-M_{o}^{2} \\
-\left(E+M_{o}\right) V(r)
\end{array}\right) \psi(r, \theta, \varphi)=0
$$

and here we have set $V(r) \rightarrow(1 / 2) V(r)$.

Accordingly, it would be natural to scale the potential term in (6), so that the nonrelativistic energy is reproduced [18]. The new wave function that is used to get the approximate form of Klein-Gordon equation is given [11]:

$$
\psi(r, \theta, \varphi)=\left(1+2 \alpha_{M L} \Delta\right) \phi(r, \theta, \varphi)
$$

Equation (7) is modification of (19) in [11]. The modification is proposed to eliminate quadratic of Laplacian factor such that we get (20) in [11]. By substituting (7) into (6), we obtain

$$
\left[-\Delta-\frac{\left(\begin{array}{c}
E^{2}-M_{o}^{2} \\
-\left(E+M_{o}\right) V(r)
\end{array}\right)}{\left(1+2 \alpha_{M L}\left(\begin{array}{c}
E^{2}-M_{o}^{2} \\
-\left(E+M_{o}\right) \\
V(r)
\end{array}\right)\right)}\right] \phi(r, \theta, \varphi)=0
$$

Equation (8) is the minimal length formalism in KleinGordon equation within the approximate form. The component $\Delta^{3}$ is eliminated due to the value of $\alpha_{M L}^{2}$ which goes to zero, and value of $\alpha_{M L}$ is very small. Here, we have used the properties that $\Delta$ is scalar differential operator. If $\Delta$ operates to scalar fields $\phi$ at a point $(r, \theta, \varphi), \Delta \phi$ will result in another scalar field. Here, $\Delta \phi$ is also called scalar Laplacian. Inserting (7) into (6) leads us to do the multiplication operation among Laplacian. By using the property that the multiplication operation is commutative for scalar differential operator with constant coefficient, then the multiplication operator among Laplacian also has commutative properties. Therefore, here we can eliminate $\Delta^{2}$ when (7) is inserted into (6). To get simple solution of (8), binomial expansion is used for small $\alpha_{M L}$; then (8) becomes

$$
\begin{aligned}
& \Delta \phi(r, \theta, \varphi) \\
& +\left[\begin{array}{c}
\left(E^{2}-M_{o}^{2}\right) \\
-\left(E+M_{o}\right) V(r) \\
-2 \alpha_{M L}\left(\begin{array}{c}
E^{2}-M_{o}^{2} \\
-\left(E+M_{o}\right) V(r)
\end{array}\right)^{2}
\end{array}\right] \phi(r, \theta, \varphi)=0
\end{aligned}
$$

Equation (9) is obtained by setting $\alpha_{M L}{ }^{2}$ which goes to zero, so $\alpha_{M L}{ }^{2}$ is ignored. Applying spherical Laplacian operator, as

$$
\begin{aligned}
\Delta= & \frac{1}{r^{2}} \frac{\partial}{\partial r} r^{2} \frac{\partial}{\partial r} \\
& +\frac{1}{r^{2}}\left(\frac{1}{\sin \theta} \frac{\partial}{\partial \theta} \sin \theta \frac{\partial}{\partial \theta}+\frac{1}{\sin ^{2} \theta} \frac{\partial^{2}}{\partial \varphi^{2}}\right)
\end{aligned}
$$

into (9), we use variable separable method by setting the new wave function $\phi(r, \theta, \varphi)=R(r) \Theta(\theta) \Phi(\varphi)$, and we have a polar part and radial part of Klein-Gordon equation in the presence of minimal length. The polar part is given:

$$
-\left[\begin{array}{c}
\frac{1}{\Theta(\theta)} \frac{1}{\sin \theta} \frac{\partial}{\partial \theta} \sin \theta \frac{\partial \Theta(\theta)}{\partial \theta} \\
+\frac{1}{\Phi(\varphi)} \frac{1}{\sin ^{2} \theta} \frac{\partial^{2} \Phi(\varphi)}{\partial \varphi^{2}}
\end{array}\right]=\lambda
$$


and the radial part is as follows:

$$
\begin{aligned}
& \frac{1}{r^{2}} \frac{\partial}{\partial r} r^{2} \frac{\partial R(r)}{\partial r} \\
& \left.+\left[\begin{array}{c}
\left(\begin{array}{c}
E^{2}-M_{o}^{2} \\
-\left(E+M_{o}\right) V(r)
\end{array}\right) \\
\left.-2 \alpha_{M L}\left(\begin{array}{c}
\left(E^{2}-M_{o}^{2}\right)^{2} \\
-2\left(E^{2}-M_{o}^{2}\right) \\
\left(E+M_{o}\right) V(r) \\
+\left(E+M_{o}\right)^{2} V^{2}(r)
\end{array}\right)\right] R(r)
\end{array}\right]\right) \\
& \quad=\frac{\lambda}{r^{2}} R(r)
\end{aligned}
$$

where $\lambda$ is a constant of variable separable method which corresponds to angular momentum $(L)$. By applying $R(r)=$ $U_{H}(r) / r$ and $\lambda=L(L+1)$ into (12), it yields

$$
\begin{aligned}
& \frac{d^{2} U_{H}(r)}{d r^{2}}-\frac{L(L+1)}{r^{2}} U_{H}(r) \\
& +\left[\begin{array}{c}
\left(\begin{array}{c}
E^{2}-M_{o}^{2} \\
-\left(E+M_{o}\right) V(r)
\end{array}\right) \\
\left(E^{2}-M_{o}^{2}\right)^{2} \\
-2 \alpha_{M L}\left(\begin{array}{c}
2 \\
\left.-M_{o}^{2}\right)\left(E+M_{o}\right) V(r) \\
+\left(E+M_{o}\right)^{2} V^{2}(r)
\end{array}\right)
\end{array}\right] U_{H}(r) \\
& =0
\end{aligned}
$$

Equation (13) is the minimal length formalism in KleinGordon equation with Hulthen potential in the form of onedimensional Schrodinger-like equation.

\section{Asymptotic Iteration Method}

Asymptotic Iteration Method is method to solve the secondorder differential equation in form [19-21]

$$
y_{n}^{\prime \prime}(u)=\lambda_{0}(u) y_{n}^{\prime}(u)+s_{0}(u) y_{n}(u)
$$

where $\lambda_{0}(u) \neq 0$ and $s_{0}(u)$ are the coefficients of a differential equation and $n$ is a quantum number. To obtain solution, we derive (14).

$$
y_{n}{ }^{z+1}(u)=\lambda_{z-1}(u) y_{n}^{\prime}(u)+s_{z-1}(u) y_{n}(u)
$$

and here

$$
\begin{aligned}
\lambda_{z}(u) & =\lambda_{z-1}^{\prime}(u)+s_{z-1}(u)+\lambda_{0}(u) \lambda_{z-1}(u) \\
s_{z}(u) & =s_{z-1}^{\prime}(u)+s_{0}(u) \lambda_{z-1}(u), \\
z & =1,2,3, \ldots
\end{aligned}
$$

The eigenvalue is obtained from the quantization condition which is given by

$$
\Delta_{z}(u)=\lambda_{z}(u) s_{z-1}(u)-\lambda_{z-1}(u) s_{z}(u)=0
$$

To obtain the wave function, (14) is reduced into the formalism, as follows

$$
y_{n}^{\prime \prime}(u)=\left\{\begin{array}{c}
2\left(\frac{a u^{N+1}}{1-b u^{N+2}}-\frac{t+1}{u}\right) y_{n}^{\prime}(u) \\
-\frac{w u^{N}}{1-b u^{N+2}} y_{n}(u)
\end{array}\right\}
$$

Equation (18) is one-dimensional Schrodinger-like equation that has solution which is expressed in hypergeometric term, given as

$$
\begin{gathered}
y_{n}(u)=(-1)^{n} C^{\prime}(N+2)^{n}(\sigma)_{n} \\
{ }_{2} F_{1}\left(-n, \rho+n ; \sigma ; b u^{N+2}\right)
\end{gathered}
$$

where

$$
\begin{aligned}
(\sigma)_{n} & =\frac{\Gamma(\sigma+n)}{\Gamma(\sigma)} ; \\
\sigma & =\frac{2 t+N+3}{N+2} ; \\
\rho & =\frac{(2 t+1) b+2 a}{(N+2) b}
\end{aligned}
$$

$C^{\prime}$ is normalization constant and ${ }_{2} F_{1}$ is a hypergeometric function. The unnormalized wave functions of Klein-Gordon equation are obtained by using (19)-(20) [19-21].

\section{Hulthen Potential}

The Hulthen potential is one of short range potentials in physics. The Hulthen potential has been used in particle physics, atomic physics, nuclear physics, solid-state physics, and chemical physics. The Hulthen wave functions have been used in solid-state physics problems [22]. The Hulthenlike wave functions have been found to investigate atomic problems [22]. The general Hulthen potential is given by [23]

$$
V(r)=-V_{H} \omega_{H} \frac{e^{-2 \omega_{H} r}}{1-e^{-2 \omega_{H} r}}
$$

where $\omega_{H}$ is a screening parameter and $V_{H}$ is potential depth. The value of screening potential is 0.025 for low screening and 0.15 for high screening [22]. To get simple solution, (21) was changed in hyperbolic trigonometric term [23], given as

$$
V(r)=\frac{V_{H} \omega_{H}}{2}\left(1-\operatorname{coth} \omega_{H} r\right)
$$

By setting $V_{H}=7$ and $\omega_{H}=0.1$, the visualization of Hulthen potential is expressed in Figure 1.

Figure 1 shows the visualization of Hulthen potential in $r$ function. The Hulthen potential for different value of $r$ is approximately from 0 until $0.05 \mu / \mathrm{eV}$ (natural unit). The Hulthen potential has negative value in a very small value of $r$, while the Hulthen potential inclines to be constant for higher value of $r$. 


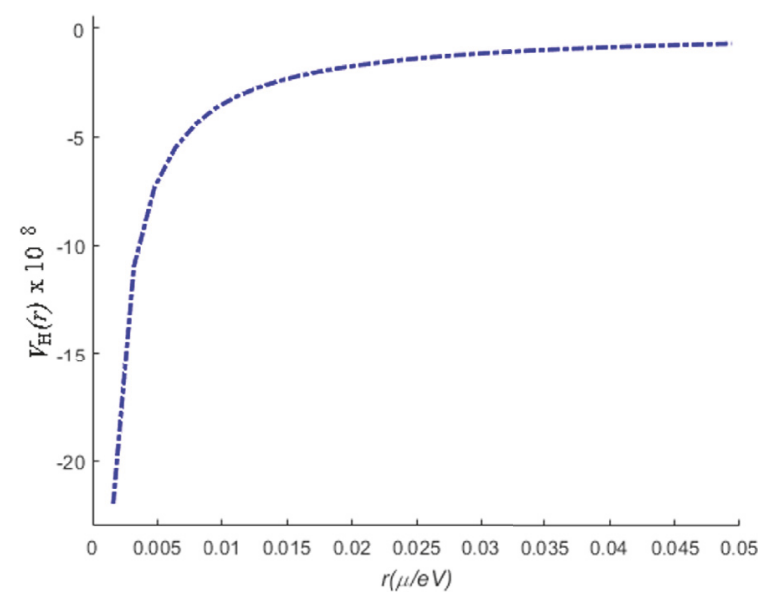

FIgURE 1: The visualization of Hulthen potential.

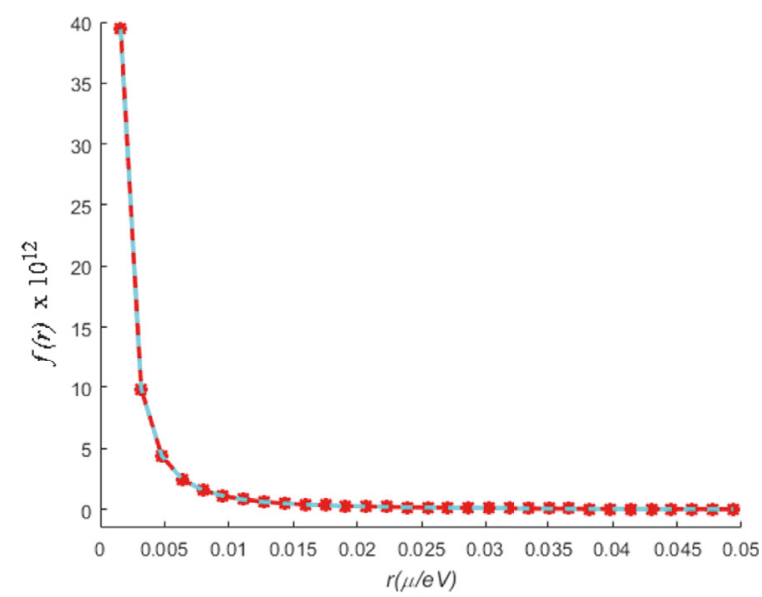

Figure 2: The visualization of approximation, with $\omega_{H}=0.1$ and value of $r$ from 0 until $0.05 \mu / \mathrm{eV}$ (natural unit).

\section{Result and Discussion}

Equation (13) can not be solved exactly unless we use the approximation to the function of $1 / r^{2}$. The approximation of function $1 / r^{2}$ is given as [23]

$$
\frac{1}{r^{2}}=\frac{\omega_{H}^{2}}{\sinh ^{2} \omega_{H} r}=f(r)
$$

for small value of $\omega_{H}$ or $\omega_{H} r \ll 1$. The visualization of that approximation is expressed in Figure 2.

Figure 2 shows the red line as a function of $1 / r^{2}$ and light blue line as a function of $\omega_{H}^{2} / \sinh ^{2}\left(\omega_{H} r\right)$. It is seen that the two lines overlap with each other; then the centrifugal term $1 / r^{2}$ is approximated by $\omega_{H}^{2} / \sinh ^{2}\left(\omega_{H} r\right)$ also as in [23].To obtain the exact solution of (13) the approximate term of $1 / r^{2}$ in (23) is inserted into (13) and together with (22); then we get

$$
\left.\left.\begin{array}{l}
\frac{d^{2} U_{H}(r)}{d r^{2}}-\frac{\left(\omega_{H}^{2} L(L+1)+\alpha_{M L}\left(E+M_{o}\right)^{2}\left(\left(V_{H} \omega_{H}\right)^{2} / 2\right)\right)}{\sinh ^{2} \omega_{H} r} U_{H}(r) \\
+\left[\begin{array}{c}
+\left(\begin{array}{c}
\left(E+M_{o}\right) \frac{V_{H} \omega_{H}}{2}+\alpha_{M L}\left(E+M_{o}\right)^{2}\left(V_{H} \omega_{H}\right)^{2} \\
-2 \alpha_{M L}\left(E^{2}-M_{o}^{2}\right)\left(E+M_{o}\right) V_{H} \omega_{H}
\end{array}\right) \operatorname{coth} \omega_{H} r \\
+\left(\begin{array}{c}
\left(E^{2}-M_{o}^{2}\right)-\left(E+M_{o}\right) \frac{V_{H} \omega_{H}}{2} \\
+2 \alpha_{M L}\left(E^{2}-M_{o}^{2}\right)\left(E+M_{o}\right) V_{H} \omega_{H} \\
-\alpha_{M L}\left(E+M_{o}\right)^{2}\left(V_{H} \omega_{H}\right)^{2}-2 \alpha_{M L}\left(E^{2}-M_{o}^{2}\right)^{2}
\end{array}\right)
\end{array}\right] U_{H}(r)=0
\end{array}\right]\right]^{2}
$$


Equation (24) is the Klein-Gordon equation with the minimal length for Hulthen potential which can be rewritten as

$$
\begin{aligned}
& \frac{d^{2} U_{H}(r)}{d r^{2}} \\
& \quad-\left[\frac{v_{P H}\left(v_{P H}-1\right)}{\sinh ^{2} \omega_{H} r}-2 q_{P H} \operatorname{coth} \omega_{H} r+\kappa_{P H}^{2}\right] U_{H}(r) \\
& =0
\end{aligned}
$$

with

$$
\begin{aligned}
& v_{P H}\left(v_{P H}-1\right) \\
& =\left(\omega_{H}^{2} L(L+1)+\alpha_{M L}\left(E+M_{o}\right)^{2} \frac{\left(V_{H} \omega_{H}\right)^{2}}{2}\right) \\
& 2 q_{P H} \\
& =\left(\begin{array}{c}
\left(E+M_{o}\right) \frac{\left(V_{H} \omega_{H}\right)}{2}+\alpha_{M L}\left(E+M_{o}\right)^{2}\left(V_{H} \omega_{H}\right)^{2} \\
-2 \alpha_{M L}\left(E^{2}-M_{o}^{2}\right)\left(E+M_{o}\right)\left(V_{H} \omega_{H}\right)
\end{array}\right) \\
& -\kappa_{P H}^{2} \\
& =\left(\begin{array}{c}
\left(E^{2}-M_{o}^{2}\right)-\left(E+M_{o}\right) \frac{\left(V_{H} \omega_{H}\right)}{2} \\
+2 \alpha_{M L}\left(E^{2}-M_{o}^{2}\right)\left(E+M_{o}\right)\left(V_{H} \omega_{H}\right) \\
-\alpha_{M L}\left(E+M_{o}\right)^{2}\left(V_{H} \omega_{H}\right)^{2}-2 \alpha_{M L}\left(E^{2}-M_{o}^{2}\right)^{2}
\end{array}\right)
\end{aligned}
$$

Equation (25) is second-order differential equation that will be reduced to hypergeometric differential equation type; by letting coth $\omega_{H} r=1-2 z$, we get

$$
\begin{gathered}
z(1-z) \frac{d^{2} U_{H}(r)}{d z^{2}}+(1-2 z) \frac{d U_{H}(r)}{d z} \\
+\left[v_{P H}^{\prime}\left(v_{P H}^{\prime}-1\right)-\frac{-2 q_{P H}^{\prime}+\kappa_{P H}^{\prime 2}}{4 z}\right. \\
\left.+\frac{2 q_{P H}^{\prime}+\kappa_{P H}^{\prime 2}}{4(1-z)}\right] U_{H}(r)=0
\end{gathered}
$$

and then we set

$$
\begin{aligned}
2 q_{P H}^{\prime} & =\frac{2 q_{P H}}{\omega_{H}^{2}} ; \\
\kappa_{P H}^{\prime 2} & =\frac{\kappa_{P H}^{2}}{\omega_{H}^{2}} \\
v_{P H}^{\prime}\left(v_{P H}^{\prime}-1\right) & =\frac{v_{P H}\left(v_{P H}-1\right)}{\omega_{H}^{2}} ; \\
-2 q_{P H}^{\prime}+\kappa_{P H}^{\prime 2} & =4 \alpha_{P H}^{2} ; \\
2 q_{P H}^{\prime}+\kappa_{P H}^{\prime 2} & =4 \beta_{P H}^{2}
\end{aligned}
$$

By setting $U_{H}(r)=z^{\alpha_{P H}}(1-z)^{\beta_{P H}} f_{H}(z)$, then (31) is reduced to AIM-type differential equation that is similar to (14).

$$
\begin{aligned}
& f_{H}^{\prime \prime}(z)+\left[\frac{\left(2 \alpha_{P H}+1\right)-\left(2 \alpha_{P H}+2 \beta_{P H}+2\right) y}{z(1-z)}\right] f_{H}^{\prime}(z) \\
& +\left[\frac{v_{P H}^{\prime}\left(v_{P H}^{\prime}-1\right)-\left(\alpha_{P H}+\beta_{P H}\right)\left(\alpha_{P H}+\beta_{P H}+1\right)}{z(1-z)}\right] \\
& \cdot f_{H}(z)=0
\end{aligned}
$$

By comparing (14) and (32), we have

$$
\begin{aligned}
& \lambda_{0_{P H}}=\frac{-\left(2 \alpha_{P H}+1\right)}{z}+\frac{\left(2 \beta_{P H}+1\right)}{1-z} \\
& s_{0_{P H}} \\
& =\left[\begin{array}{c}
\frac{\left(\alpha_{P H}+\beta_{P H}\right)\left(\alpha_{P H}+\beta_{P H}+1\right)-v_{P H}^{\prime}\left(v_{P H}^{\prime}-1\right)}{z} \\
+\frac{\left(\alpha_{P H}+\beta_{P H}\right)\left(\alpha_{P H}+\beta_{P H}+1\right)-v_{P H}^{\prime}\left(v_{P H}^{\prime}-1\right)}{(1-z)}
\end{array}\right]
\end{aligned}
$$

To obtain eigenvalue, we use (15)-(17) and (33)-(34), which yields

$$
\begin{aligned}
& v_{P H}^{\prime}\left(v_{P H}^{\prime}-1\right) \\
& \quad=\left(\alpha_{P H}+\beta_{P H}+n\right)\left(\alpha_{P H}+\beta_{P H}+(n+1)\right)
\end{aligned}
$$

By inserting (27)-(28) and (30) into (35), we obtain the relativistic energy equation Klein-Gordon equation with minimal length for Hulthen potential, as follows:

$$
\begin{aligned}
& \left(E^{2}-M_{o}^{2}\right) \\
& =\left\{\begin{array}{c}
{\left[\omega_{H}^{2}\left[\begin{array}{c}
\left(\sqrt{v_{P H}^{\prime}\left(v_{P H}^{\prime}-1\right)+\frac{1}{4}}-n-\frac{1}{2}\right)^{2} \\
4\left(\omega_{H}^{2} \sqrt{v_{P H}^{\prime}\left(v_{P H}^{\prime}-1\right)+1 / 4}-n-1 / 2\right)^{2}
\end{array}\right]\right\}} \\
+\left(E+M_{o}\right) \frac{V_{H}}{2}+\tau_{P H}
\end{array}\right]
\end{aligned}
$$

where

$$
\begin{aligned}
v_{P H}^{\prime} & =\frac{1}{2} \\
+ & \sqrt{\frac{\left(\omega_{H}^{2} L(L+1)+\alpha_{M L}\left(E+M_{o}\right)^{2}\left(\left(V_{H} \omega_{H}\right)^{2} / 2\right)\right)}{\omega_{H}^{2}}+\frac{1}{4}} \\
\zeta_{P H} & =\left(\begin{array}{c}
\left(E+M_{o}\right) \frac{\left(V_{H} \omega_{H}\right)}{2}+\alpha_{M L}\left(E+M_{o}\right)^{2}\left(V_{H} \omega_{H}\right)^{2} \\
-2 \alpha_{M L}\left(E^{2}-M_{o}^{2}\right)\left(E+M_{o}\right)\left(V_{H} \omega_{H}\right)
\end{array}\right)
\end{aligned}
$$

$$
z(1-z) \frac{d^{2} U_{H}(r)}{d z^{2}}+(1-2 z) \frac{d U_{H}(r)}{d z}
$$


TABLE 1: Relativistic energy with $V_{H}=7, \omega_{H}=0.1, M_{o}=1$, and $L=0$.

\begin{tabular}{ccccc}
\hline$n$ & \multicolumn{4}{c}{$E(\mathrm{eV})$} \\
& $\alpha_{M L}=0$ & $\alpha_{M L}=0.001$ & $\alpha_{M L}=0.01$ & $\alpha_{M L}=0.1$ \\
\hline 0 & -1.9770 & -1.9654 & -1.8759 & -1.5259 \\
1 & -1.8629 & -1.8537 & -1.7801 & -1.4725 \\
2 & -1.7473 & -1.7402 & -1.6818 & -1.4171 \\
3 & -1.6298 & -1.6248 & -1.5808 & -1.3594 \\
4 & -1.5102 & -1.5071 & -1.4767 & -1.2990 \\
5 & -1.3879 & -1.3865 & -1.3690 & -1.2357 \\
\hline
\end{tabular}

$$
\tau_{P H}=\left[\begin{array}{c}
\alpha_{M L}\left(E+M_{o}\right)^{2}\left(V_{H} \omega_{H}\right)^{2} \\
+2 \alpha_{M L}\left(E^{2}-M_{o}^{2}\right)^{2} \\
-2 \alpha_{M L}\left(E^{2}-M_{o}^{2}\right)\left(E+M_{o}\right)\left(V_{H} \omega_{H}\right)
\end{array}\right]
$$

and $n$ is quantum number. Equation (36) is relativistic energy equation of the minimal length formalism in Klein-Gordon equation with Hulthen potential. The relativistic energies were calculated numerically by using Matlab software. The results of relativistic energies are listed in Table 1.

Table 1 shows that the presence of minimal length and Hulthen potential in Klein-Gordon equation gives influence to the relativistic energy value. The relativistic energy value without minimal length parameter $\left(\alpha_{M L}=0\right)$ is lower than the relativistic energy value with minimal length parameter. Then, the relativistic energy value increases for the larger minimal length parameter and for the larger quantum number $(n)$. The influence of Hulthen potential gives negative value in the relativistic energy value. If we set $\alpha_{M L}=$ 0 , without the presence of the minimal length parameter in relativistic energy equation (36), it was reduced to the relativistic energy equation which is in agreement with [5]. In [5] it was shown that the relativistic energy equation for the Klein-Gordon equation for Hulthen potential without the minimal length depends on the squared of quantum number (n).

To get the unnormalized wave function, we used (19)(20), so we have

$$
\begin{aligned}
& N_{P H}=-1 ; \\
& a_{P H}=\left(\beta_{P H}+\frac{1}{2}\right) ; \\
& b_{P H}=1 ; \\
& t_{P H}=\left(\alpha_{P H}-\frac{1}{2}\right) ; \\
& \sigma_{P H}=2 \alpha_{P H}+1 ; \\
& \rho_{P H}=2 \alpha_{P H}+2 \beta_{H}+1
\end{aligned}
$$

Inserting (40) into (19) yields

$$
\begin{aligned}
& f_{H}(z) \\
& \quad=\left[\begin{array}{c}
(-1)^{n} C^{\prime}(1)^{n}\left(2 \alpha_{P H}+1\right)_{n} \\
{ }_{2} F_{1}\left(-n, 2 \alpha_{P H}+2 \beta_{P H}+1+n, 2 \alpha_{P H}+1, z\right)
\end{array}\right]
\end{aligned}
$$

Equation (41) is substituted into $U_{H}(r)=z^{\alpha_{P H}}(1-z)^{\beta_{P H}} f_{H}(z)$, so we have the unnormalized wave function given as

$$
\begin{aligned}
U_{H}(r) & \\
\quad= & {\left[\begin{array}{c}
z^{\alpha_{P H}}(1-z)^{\beta_{P H}}(-1)^{n} C^{\prime}(1)^{n}\left(2 \alpha_{P H}+1\right)_{n} \\
{ }_{2} F_{1}\left(-n, 2 \alpha_{P H}+2 \beta_{P H}+1+n, 2 \alpha_{P H}+1, z\right)
\end{array}\right] }
\end{aligned}
$$

By applying coth $\omega_{H} r=1-2 z$ in (42), we obtain the unnormalized wave function of minimal length formalism in Klein-Gordon equation, given as

$$
\begin{aligned}
& U_{H_{0}}(z)=C^{\prime}\left(\frac{1-\operatorname{coth} \omega_{H} r}{2}\right)^{\alpha_{P H}}\left(\frac{1+\operatorname{coth} \omega_{H} r}{2}\right)^{\beta_{P H}} \\
& U_{H_{1}}(z)=\left\{\begin{array}{c}
-C^{\prime}\left(\frac{1-\operatorname{coth} \omega_{H} r}{2}\right)^{\alpha_{P H}}\left(\frac{1+\operatorname{coth} \omega_{H} r}{2}\right)^{\beta_{P H}}\left(2 \alpha_{P H}+1\right) \\
{\left[1+\frac{(-1)\left(2 \alpha_{P H}+2 \beta_{P H}+2\right)\left(\left(1-\operatorname{coth} \omega_{H} r\right) / 2\right)}{\left(2 \alpha_{P H}+1\right) 1 !}\right]}
\end{array}\right\} \\
& U_{H_{2}}(r)=\left\{\begin{array}{c}
-C^{\prime}\left(\frac{1-\operatorname{coth} \omega_{H} r}{2}\right)^{\alpha_{P H}}\left(\frac{1+\operatorname{coth} \omega_{H} r}{2}\right)^{\beta_{P H}}\left(\begin{array}{c}
\left(2 \alpha_{P H}+1\right) \\
\left(2 \alpha_{P H}+2\right)
\end{array}\right) \\
{\left[\begin{array}{c}
1+\frac{\left(\begin{array}{c}
(-1)\left(2 \alpha_{P H}+2 \beta_{P H}+2\right) \\
\left(\left(1-\operatorname{coth} \omega_{H} r\right) / 2\right)
\end{array}\right)}{\left(2 \alpha_{P H}+1\right) 1 !} \\
+\frac{\left(\begin{array}{c}
(-2)(-1)\left(2 \alpha_{P H}+2 \beta_{P H}+3\right) \\
\left(2 \alpha_{P H}+2 \beta_{P H}+4\right)\left(\left(1-\operatorname{coth} \omega_{H} r\right) / 2\right)^{2}
\end{array}\right)}{\left(2 \alpha_{P H}+1\right)\left(2 \alpha_{P H}+2\right) 2 !}
\end{array}\right]}
\end{array}\right\}
\end{aligned}
$$

Equations (43), (44), and (45) are the unnormalized wave functions $n=0$ for ground state, $n=1$ for energy level 1 , and $n=2$ for energy level 2 , respectively. The visualization of the unnormalized wave functions is shown in Figures 3 and 4. 


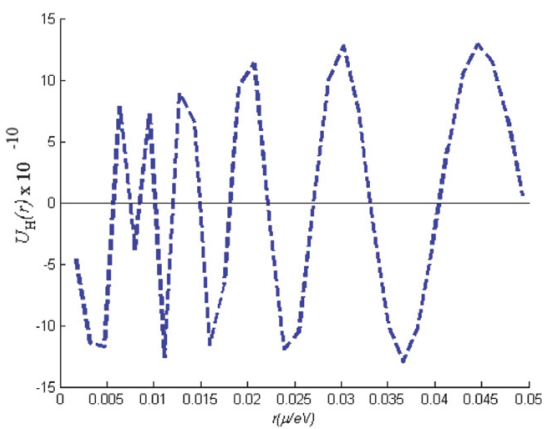

(a)

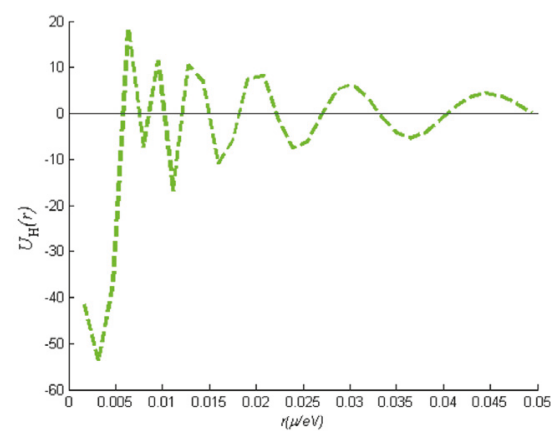

(b)

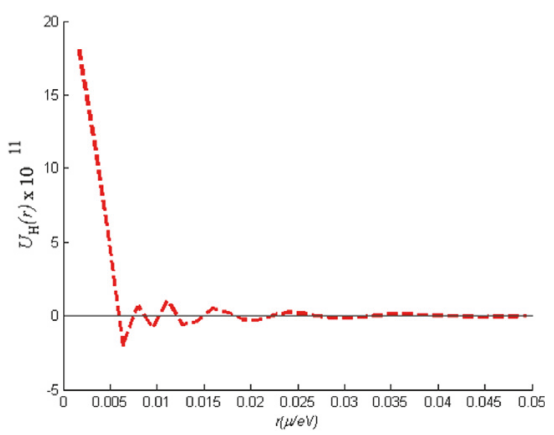

(c)

Figure 3: The unnormalized wave functions with $\alpha_{M L}=0$ for (a) $n=0$, (b) $n=1$, and (c) $n=2$.

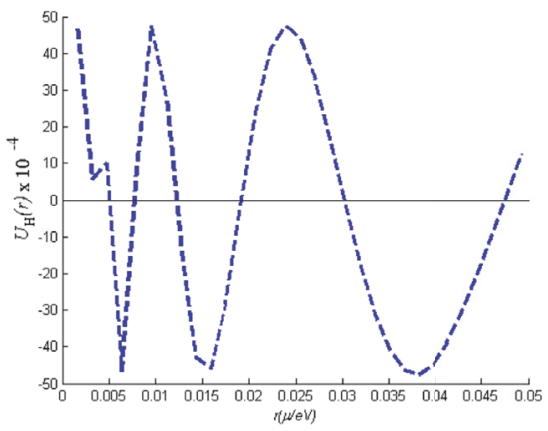

(a)

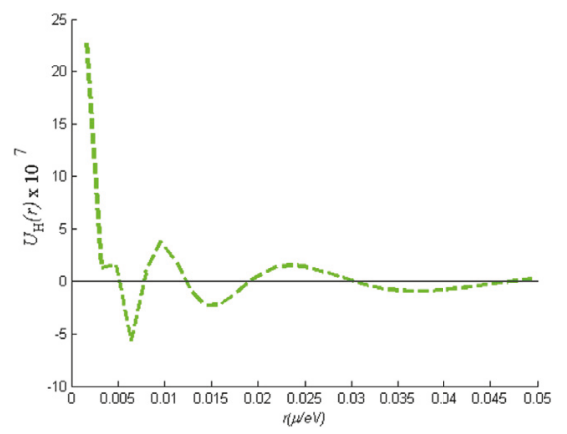

(b)

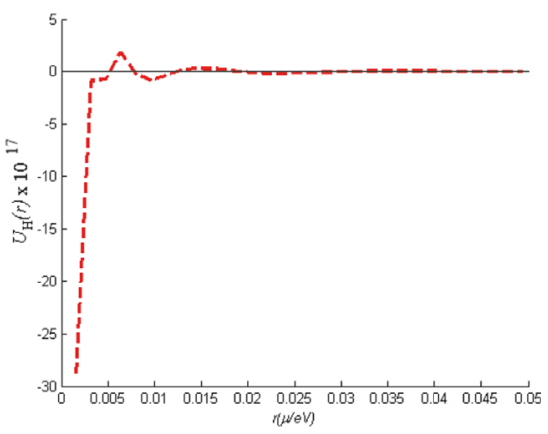

(c)

FIGURE 4: The unnormalized wave functions with $\alpha_{M L}=0.1$ for (a) $n=0$, (b) $n=1$, and (c) $n=2$.

By inspecting Figures 3 and 4, we can see that the influence of minimal length parameter increases the amplitude of the unnormalized wave function.

\section{Conclusion}

The investigation of the minimal length formalism in the Klein-Gordon equation is obtained by approximate solution. The minimal length in Klein-Gordon equation for Hulthen potential is solved using Asymptotic Iteration Method. The Asymptotic Iteration Method is used to obtain the relativistic energy and unnormalized wave functions. The results show that the relativistic energies value increases for the larger minimal length parameter and for the larger quantum number (n). Then, the influence of minimal length parameter exerts effect in increasing the amplitude value of the unnormalized wave functions.

\section{Data Availability}

All data used to support the findings of this study are included within the article.

\section{Conflicts of Interest}

The authors declare that they have no conflicts of interest.

\section{Acknowledgments}

This research was partly supported by Ministry of Research, Technology, and Higher Education with Contract no. 089/SP2H/LT/DRPM/2018.

\section{Supplementary Materials}

To solve the iteration equation and to do the numerical calculation, other software can be used such as Maple, Mathematica, and Octave. (Supplementary Materials)

\section{References}

[1] A. D. Antia, A. N. Ikot, E. E. Ituen, and I. O. Akpan, "Bound state solutions of the Klein - Gordon equation for deformed Hulthen potential with position dependent mass," Sri Lankan Journal of Physics, vol. 13, no. 1, pp. 27-40, 2012.

[2] M. C. Zhang and Z. B. Wang, "Exact solutions of the KleinGordon equation with Makarov potential and a recurrence relation," Chinese Physics, vol. 16, no. 7, article 1863, 2007.

[3] S. Ortakaya, "Exact solutions of the klein-Gordon equation with ring-shaped oscillator potential by using the Laplace integral transform," Chinese Physics B, vol. 21, no. 7, 2012.

[4] H. Hassanabadi, S. Zarrinkamar, and H. Rahimov, "Approximate solution of D-dimensional Klein - Gordon equation with hulthén-type potential via SUSYQM," Communications in Theoretical Physics, vol. 56, no. 3, pp. 423-428, 2011. 
[5] A. N. Ikot, L. E. Akpabiol, and E. J. Uwah, "Bound state solutions of the klein gordon equationwith the hulthen potential," Electronic Journal of Theoretical Physics, vol. 8, no. 25, pp. 225232, 2011.

[6] D. A. Nugraha, A. Suparmi, C. Cari, and B. N. Pratiwi, "Asymptotic iteration method for solution of the Kratzer potential in D-dimensional Klein-Gordon equation," Journal of Physics: Conference Series, vol. 820, 2017.

[7] D. A. Nugraha, A. Suparmi, and C. Cari, "Asymptotic Iteration Method foranalytical solution of Klein Gordon equation for Trigonometric Pöschl-Teller potential in D dimensions," Journal of Theoretical and Applied Physics, vol. 795, no. 1, p. 42, 2017.

[8] A. Poszwa, "Relativistic generalizations of the quantum harmonic oscillator," Acta Physica Polonica A, vol. 126, no. 6, pp. 1226-1234, 2014.

[9] L. Garay, "Quantum gravity and minimum length," International Journal of Modern Physics A, vol. 10, no. 2, pp. 145-165, 1995.

[10] A. Tilbi, M. Merad, and T. Boudjedaa, "Particles of spin zero and $1 / 2$ in electromagnetic field with confining scalar potential in modified heisenberg algebra," Few-Body Systems, vol. 56, no. 2-3, pp. 139-147, 2015.

[11] M. Chabab, A. El Batoul, A. Lahbas, and M. Oulne, "On $\gamma$-rigid regime of the Bohr-Mottelson Hamiltonian in the presence of a minimal length," Physics Letters B, vol. 758, pp. 212-218, 2016.

[12] M. Alimohammadi and H. Hassanabadi, "Alternative solution of the gamma-rigid Bohr Hamiltonian in minimal length formalism," Nuclear Physics A, vol. 957, pp. 439-449, 2017.

[13] T. K. Jana and P. Roy, "Exact solution of the Klein-Gordon equation in the presence of a minimal length," Physics Letters A, vol. 373, no. 14, pp. 1239-1241, 2009.

[14] M. Merad, F. Zeroual, and H. Benzair, "Spinless relativistic particle in the presence of a minimal length," Electronic Journal of Theoretical Physics, vol. 6, no. 23, pp. 41-56, 2010.

[15] I. L. Elviyanti, A. Suparmi, C. Cari, D. A. Nugraha, and B. N. Pratiwi, "Solution of Klein Gordon equation for hyperbolic cotangent potential in the presence of a minimal length using Hypergeometric method," Journal of Physics: Conference Series, vol. 909, no. 1, 2017.

[16] A. Suparmi, C. Cari, and I. L. Elviyanti, "Solution of Klein Gordon equation for trigonometric cotangent potential in the presence of a minimal length using Asymptotic Iteration Method," Journal of Physics: Conference Series, vol. 909, no. 1, 2017.

[17] C. Cari, A. Suparmi, and I. L. Elviyanti, "Approximate solution for the minimal length case of klein gordon equation for trigonometric cotangent potential using asymptotic iteration method," Journal of Physics: Conf. Series, vol. 909, 2017.

[18] S. M. Ikhdair and M. Hamzavi, "Effects of external fields on a two-dimensional Klein-Gordon particle under pseudoharmonic oscillator interaction," Chinese Physics B, vol. 21, no. 11, p. 110302, 2012.

[19] H. Ciftci, R. L. Hall, and N. Saad, "Construction of exact solutions to eigenvalue problems by theasymptotic iteration method," Journal of Physics A: Mathematical and General, vol. 36, no. 47, pp. 11807-11816, 2003.

[20] B. N. Pratiwi, A. Suparmi, C. Cari, and A. S. Husein, "Asymptotic iteration method for the modified poschl-teller potential and trigonometric Scarf II non-central potential in the Dirac equation spin symmetry," Pramana-Journal of Physics, vol. 88, no. 2, 2017.
[21] S. Pramono, A. Suparmi, and C. Cari, "Relativistic energy analysis of five-dimensional q-deformed radial rosen-morse potential combined with q-deformed trigonometric scarf noncentral potential using asymptotic iteration method," Advances in High Energy Physics, vol. 2016, 2016.

[22] Y. P. Varshni, "Eigenenergies and oscillator strengths for the Hulthén potential," Physical Review A: Atomic, Molecular and Optical Physics, vol. 41, no. 9, pp. 4682-4689, 1990.

[23] L. Naderi and H. Hassanabadi, "Bohr Hamiltonian with Eckart potential for triaxial nuclei," The European Physical Journal Plus, vol. 131, no. 5, 2016. 


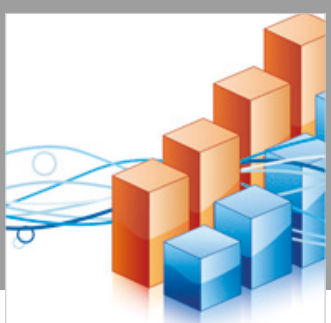

Advances in

Operations Research

\section{-n-m}
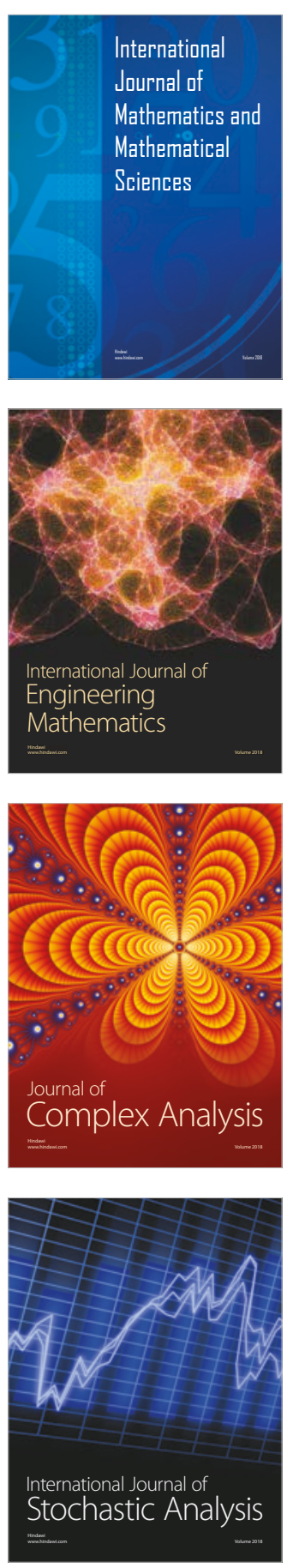
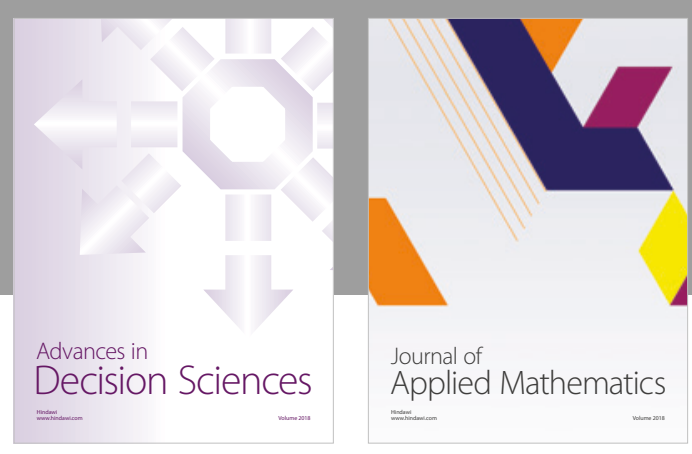

Journal of

Applied Mathematics
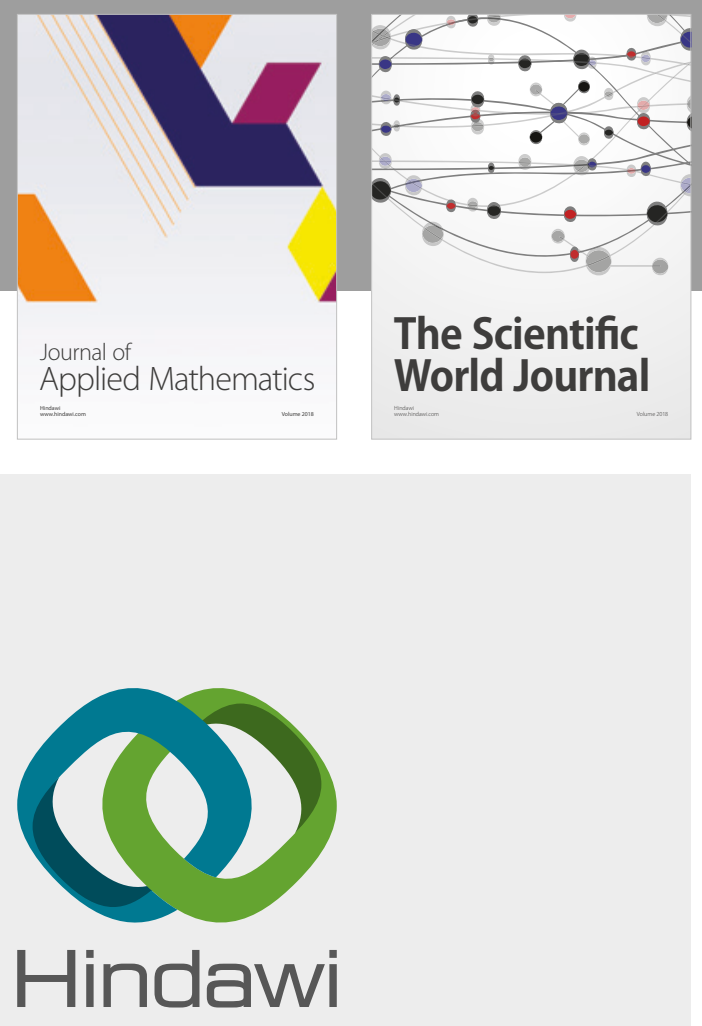

Submit your manuscripts at

www.hindawi.com

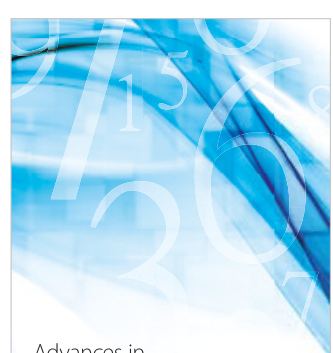

Advances in
Numerical Analysis
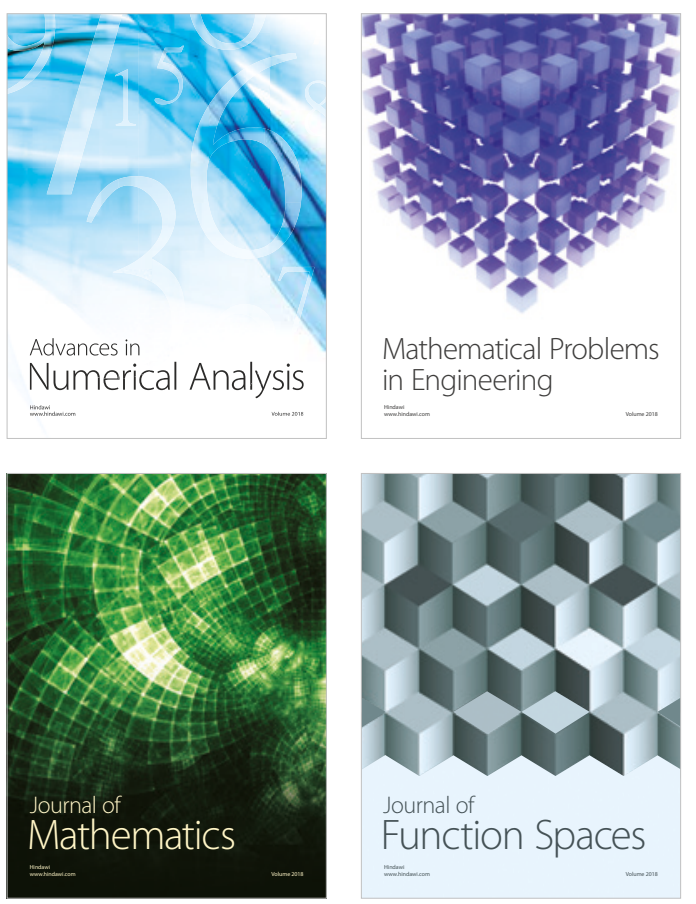

Mathematical Problems in Engineering

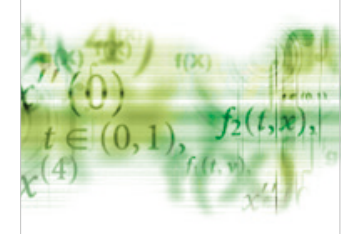

International Journal of

Differential Equations

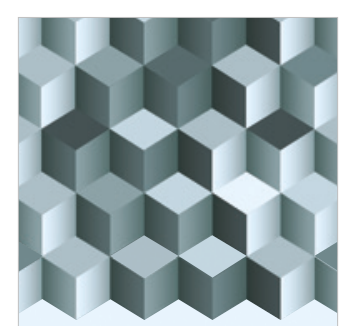

Journal of

Function Spaces

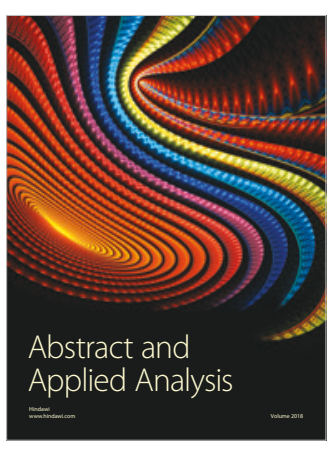

The Scientific

World Journal

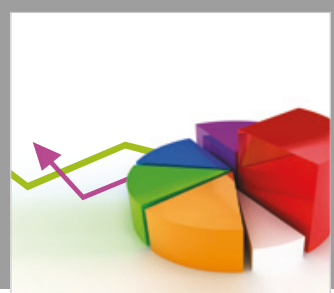

Journal of

Probability and Statistics
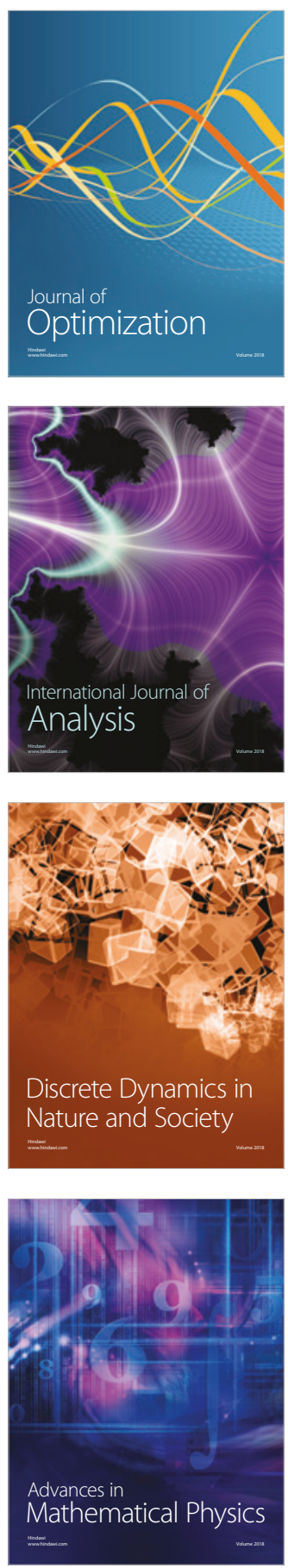